\title{
Mantle cell lymphoma: pathologic and immunophenotyping study of 50 cases and correlation with survival-a single institute experience
}

\author{
Ahmad Monabati $^{1} \cdot$ Akbar Safaei $^{1}$ - Maral Mokhtari ${ }^{1}$ - Samaneh Boroumand-Noughabi ${ }^{1} \cdot$ Marjan Faghih $^{1}$. \\ Pardis Nematollahi ${ }^{1}$ (iD
}

Received: 2 April 2020 / Accepted: 19 May 2020 / Published online: 29 May 2020

(C) Springer-Verlag GmbH Germany, part of Springer Nature 2020

\begin{abstract}
Mantle cell lymphoma (MCL) is a relatively rare lymphoma subtype. Almost all cases show common genetic abnormality $\mathrm{t}(11 ; 14)$, resulting in overexpression of cyclin D1 protein. The clinical course is usually aggressive, and the disease is mainly considered incurable but obvious clinical heterogeneity exists. Most patients experience a rapidly progressive course, and some go through an indolent course of disease. So new strategies are needed for risk stratification and may allow for a more personalized approach in the future. We retrospectively studied 50 cases of MCL which were diagnosed at a single center over about 5 years to determine clinicopathologic and immunophenotyping findings. This study focuses on (1) age, gender, and location of tumor at presentation; (2) some challenges in the diagnostic criteria; (3) cytomorphology, histomorphology, immunophenotyping, and proliferation rate; and (4) overall survival and finally relationship with demographic and pathologic findings which shows no association between survival of patients and gender, cytomorphology, histology, and location of tumor while increased mitotic activity and age are prognostically important factors. Cyclin D1 immunostaining is strictly recommended in all low-grade B cell lymphoma due to absence of CD5 expression on 20\% of confirmed MCL and also on all high-grade B cell lymphoma for avoiding overlook of blastic variant of MCL.
\end{abstract}

Keywords Mantle cell lymphoma · Antigen Ki-67 · Survival $\cdot$ Clinicopathological data $\cdot$ Iran

\section{Introduction}

Mantle cell lymphoma is a mature B cell neoplasm with a male predominance and median age of about 60 years, usually composed of monomorphic small to medium-sized lymphoid cells with irregular nuclear contours. In more than $95 \%$ of cases, there is a CCND1 translocation [1]. MCL shows unique morphological, immunophenotyping, and molecular genetic features. According to immunophenotyping data, MCL arises from cells reside in the mantle zone of lymphoid follicles [2]. Based on molecular pathogenesis, one type of MCL which shows nodal involvement at presentation originates from the mantle zone of the lymphoid follicle and frequently is SOX11-positive. A rare type of MCL (10-20\% of all types),

Pardis Nematollahi

nematolahy@yahoo.com

1 Shiraz University of Medical Sciences, Shiraz, Iran usually is SOX11 negative, may undergo somatic hypermutation in germinal centers and mostly involves the peripheral blood (PB) and bone marrow (BM). It also shows a better prognosis than nodal type $[1,2]$. Neoplastic cells are mature B cells with strong expression of pan B markers such as $\mathrm{CD} 20$, PAX5, and aberrant expression of $\mathrm{CD} 5$, and are usually negative for $\mathrm{CD} 23$ and $\mathrm{CD} 10$. A recurring cytogenetic abnormality, $\mathrm{t}(11 ; 14)$ occurs in MCL, resulting in a rearrangement of the BCL-1 gene locus and overexpression of the cyclin D1 (CCND1/PRAD1) gene which is a cell cycle regulator that facilitates the deregulation of the cell cycle and can be detected on immunostaining [3]. Although usually presents with widespread diseases, such as generalized lymphadenopathy, bone marrow and peripheral blood involvement, splenomegaly, and even bowel infiltration [4], significant heterogeneities are in the clinical presentation of the disease. Some patients present with leukemic manifestation for the first time, others with nodal or extranodal mass infiltration like lymphoma. MCL also has a variable clinical course, some patients are severely symptomatic and show progressive diseases such as 
acute leukemia while others are indolent and need no urgent medical interventions. To different aggressive and less aggressive MCL, Ki-67 proliferation index, and MIPI (Mantle Cell Lymphoma International Prognostic Index), risk stratification is used which affects by age at presentation, serum LDH level, WBC count, and performance status $[5,6]$. The goal of this study was to evaluate clinicopathologic variations in patients with MCL and its correlation with patient's survival. The term "clinical variations" here refers to the location of tumor at presentation, and the term "pathologic variations" refers to cytomorphology and histomorphology and proliferation index of tumor which is detected by $\mathrm{Ki}-67$ immunostaining.

\section{Materials and methods}

Cases diagnosed as MCL between April 2014 and September 2019 retrieved from hematopathology archive of Shiraz University Medical Sciences as the most referral one in the South part of Iran. All samples had been evaluated by morphologic examination, flow cytometry, immunohistochemistry, and molecular cytogenetic study if indicated. A total of 50 cases in which the diagnosis was confirmed were included. Liquid samples such as bone marrow and peripheral blood were stained with the Wright method. Marrow sample immunophenotyping was performed by FACSCalibur flow cytometry on the standard four-color assay by the following panel of monoclonal antibodies: CD20/CD5/CD23/FMC7 and CD19/CD10/CD38/CD45 that used Cell Quest software for data analysis. Cyclin D1 detection was performed by immunostaining on trephine marrow biopsy. In examining tissues, biopsy specimens had been fixed in $10 \%$ buffered formalin and bone marrow decalcified in 0.5 M EDTA solution, routinely processed, sectioned at $4 \mu \mathrm{m}$, and stained with hematoxylin and eosin (H\&E). All nodal and extranodal samples were immunophenotyped by immunohistochemical staining for CD20, PAX5, CD5, CD10, CD3, CD23, cyclin D1, and $\mathrm{Ki}$-67. Immunostaining was done on first specimens manually. All aspiration, H\&E, and immunostained slides reviewed by two of the authors separately for subclassification of cytomorphology, histomorphology, evaluation of immunostaining, and proliferation index. In case of discrepancy, we achieve consensus on a multi-head microscope. Histomorphology is determined by the dominant pattern (greater than $50 \%)$ of the tumor by low power $(\times 10)$. Marrow showed diffuse, nodular, or interstitial pattern and lymph node or other lymphoid tissue showed diffuse, nodular, or mantle zone pattern. Based on cytomorphology, all tumors subcategorized to classic, small, blastic, marginal zone, and pleomorphic type [7] shown in Fig. 1. In classic type, neoplastic cells show some nuclear indentation and irregularity; in small type, nucleus is small with round nuclear border; and in marginal zone pattern, neoplastic cells show abundant clear cytoplasm such as normal monocytoid B cells. The proliferation index was assessed by counting Ki-67-positive neoplastic cells in 10 random high-power fields $(\mathrm{HPF}, \times 400)(5 \mathrm{HPF}$ in small biopsies). In each HPF, 500 cells were counted, and the average percentage of $\mathrm{Ki}-67$-positive neoplastic cells considered as proliferation rate [3]. Thirty percent of positivity has been usually accepted as a cut off for high, compared with low Ki-67 expression [4].

Laboratory and clinical data of the patients have obtained from the hospital HIS system and through communication with the patient or their relatives. No other comorbidities were found responsible for patients' death. All types received the same chemotherapy regimens except CNS prophylaxis in aggressive ones. Fourteen out of 50 cases presented with leukemic manifestation and bone marrow were examined, 26 out of 50 cases with nodal involvement, and 10 out of 50 cases with extranodal presentation ( 5 digestive tracts, 2 tonsils, one spleen, one nasopharynx, and one soft tissue). Information gathered from demographic data, location of the tumor, histomorphology, cytomorphology, immunophenotyping, proliferation rate, and months survive after diagnosis was filled in SPSS sheet for statistical analysis.

\section{Statistical analysis}

Fisher's exact test was used for checking the relationship between gender and location with two group memberships (Ki67 categories). Mann-Whitney $U$ test was used to examine mean differences in age between two groups. We used the Shapiro-Wilk test to assess normality and Levene's test for homogeneity of variances. The univariate analysis was conducted by univariate Cox regression for the influence of Ki67, age, gender, location, histomorphology, and cytomorphology on survival time. Variables with significant or borderline significant values $(P \leq .10)$ in the univariate analysis were entered into the Cox model for multiple analyses. All analyses were performed using the SPSS statistical software, version 22 and $P<0.05$ was considered to be statistically significant.

\section{Results}

In our center, MCL consisted of $4.57 \%$ (50 out of 1092 patients) of all malignant lymphoma diagnosed between April 2014 and September 2019. The median age was 62 (range, 41 to 96 years) with a mean of $64.36(\mathrm{SD}=11.99)$. The majority of the patients were male $(\mathrm{M} / \mathrm{F}=4 / 1)$ and the median follow-up time was 15 (range, 1-50) months. The overall survival was about 18 months. Fifty-two percent of patients presented with nodal, $20 \%$ with extranodal (5 digestive tracts, 2 tonsils, one spleen, one nasopharynx, and one 
Fig. 1 Mantle cell lymphoma

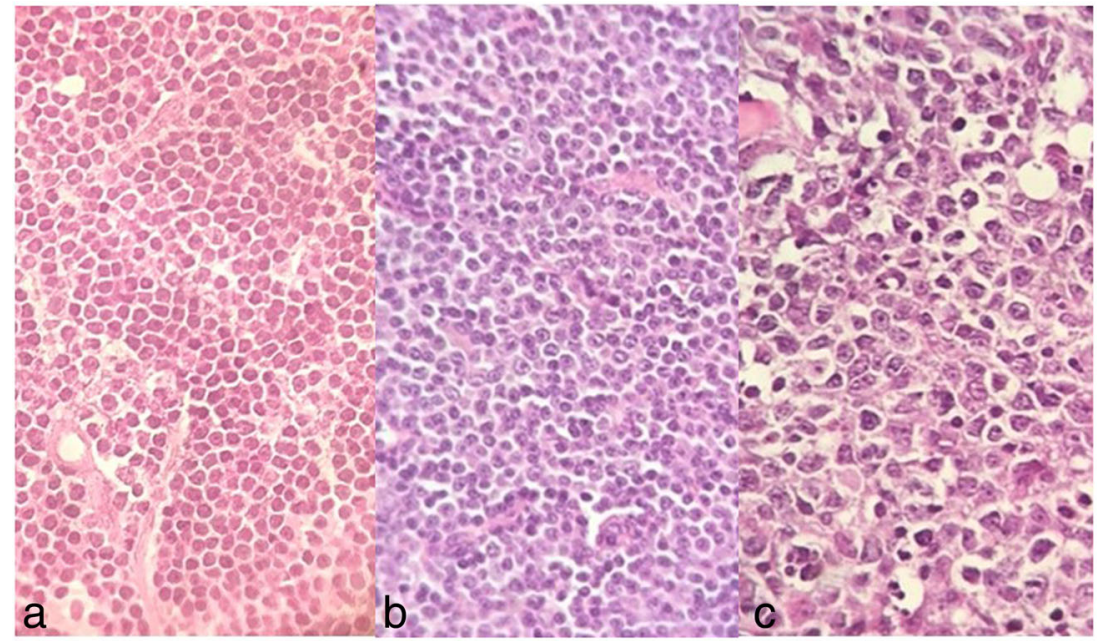

a Classic type. b Small cell Variant. C Blastoid Variant soft tissue), and $28 \%$ with leukemic involvement without evidence of lymph node involvement at the time of diagnosis. Of all patients with classic cytomorphology, 20\% were CD5 negative (all blastic and pleomorphic types expressed CD5 antigen). Clinical, demographic, and histopathologic characteristics of all patients are summarized in Table 1.
The relationship between age, gender, and location with the proliferation rate ( $\mathrm{Ki}-67$ index) is shown in Table 2. The results reveal that the 2 groups did not differ in age, gender, and location (all $P>.05$ ). The univariate and multiple Cox regression are presented in Table 3 for the influence of $\mathrm{Ki}-67$, age, gender, location,
Table 1 Clinical and demographic characteristics of all patients

\begin{tabular}{lll}
\hline Variable & Categories & Mean \pm SD or $n(\%)$ \\
\hline Age (years) & - & $64.36 \pm 11.99$ \\
Gender & Male & $40(80)$ \\
& Female & $10(20)$ \\
Location & Nodal & $26(52)$ \\
& Leukemia & $14(28)$ \\
Histomorphology of extra BM & Extra nodal & $10(20)$ \\
& Diffuse & $25(69.4)$ \\
Pattern of BM & Nodular & $10(27.8)$ \\
& Mantle zone pattern & $1(2.8)$ \\
Cytomorphology & Diffuse BM & $5(35.7)$ \\
& Nodular BM & $3(21.4)$ \\
Survival time & Interstitial BM & $6(42.9)$ \\
\hline & Classic & $34(68)$ \\
Ki-67* & Small & $7(14)$ \\
& Blastic & $5(10)$ \\
& Marginal zone & $3(6)$ \\
& Pleomorphic & $1(2)$ \\
& Ki-67 <30 & $20(40)$ \\
& Ki-67 $\geq 30$ & $16(32)$ \\
& Alive & $21(42)$ \\
& Dead & $16(32)$ \\
& - & $18.03 \pm 15.54$ \\
\hline
\end{tabular}

*Fourteen patients were missed

**Thirteen patients were missed 
Table 2 Baseline characteristics of all patients between two groups (Ki-67 <30 and Ki-67 $\geq 30)$; Mean \pm SD or $n(\%)$

\begin{tabular}{|c|c|c|c|c|c|}
\hline Variable & & Total & Ki-67 $<30(n=20)$ & $\mathrm{Ki}-67 \geq 30(n=16)$ & $P$ value \\
\hline Age (years) & & $64.36 \pm 11.99$ & $63.95 \pm 9.32$ & $67.94 \pm 13.46$ & $0.35^{*}$ \\
\hline \multirow[t]{2}{*}{ Gender } & Male & $29(80.6)$ & $14(70)$ & $15(93.8)$ & $0.1 * *$ \\
\hline & Female & $7(19.4)$ & $6(30)$ & $1(6.2)$ & \\
\hline \multirow[t]{2}{*}{ Location } & Nodal + extranodal & $30(83.3)$ & $19(95)$ & $11(68.8)$ & $0.069 * *$ \\
\hline & Leukemia & $6(16.7)$ & $1(5)$ & $5(31.2)$ & \\
\hline
\end{tabular}

*Mann-Whitney test

**Fisher's exact test

histomorphology, and cytomorphology on survival time. There are significant effects of Ki-67 and age on survival time at the level of 0.05 . There was no association between Ki-67 and histomorphologic type $(P$ value $=0.59)$ or cytomorphology $(P$ value $=0.16)$.

In the univariate analysis, $\mathrm{Ki}-67$ and age were significant risk factors for the event at the level of 0.1 (all $P \leq$ 0.1 ) (Table 3), while there was no significant difference in other variables (all $P>0.1$ ). A value of hazard ratio (HR) $=16.002>1$ in Ki-67 means that the group of Ki-67 $\geq 30$ comparing with the group of Ki-67 $<30$ has a shorter time to event, i.e., death. Also, an increase in 1 year in age has $11 \%$ (95\% CI 1.05 to 1.17 ) decrease in time to the event. In multiple Cox regression, the result revealed, $\mathrm{Ki}$ 67 and age were manifested to be risk factor for survival (Ki-67: HR $=14.71 ; 95 \% \mathrm{CI}, 1.65-130.88 ; P=.016$, and for the age, $\mathrm{HR}=1.12 ; 95 \% \mathrm{CI}, 1.03-1.22 ; P=.009)$. Therefore, Ki-67 was a significant prognostic factor after controlling age effects as covariates in the Cox regression model.

Cox Survival plot for multiple Cox regression is shown in Fig. 2. This plot is related to multiple Cox regression in $\mathrm{Ki}-67$ when age has been controlled. Survival plot for univariate Cox model of Ki-67 (all $P \leq 0.1$ ) is depicted in Fig. 3 .

\section{Discussion}

MCL consists of less than $10 \%$ of all cases of non-Hodgkin lymphoma, although is a unique lymphoma and all cases are characterized by a common genetic abnormality $\mathrm{t}(11 ; 14)$ and usually is an aggressive lymphoma but sometimes shows heterogeneous clinical behavior which may be affected by genetic and environmental factors. Actually in spite of common genetic abnormality, patients with MCL show variant cytomorphology and tumor architecture and immunophenotype which may impact prognosis and even treatment protocol. Although few studies were done on subtype distribution of lymphoma in Iran [8-10] in our knowledge, there is no specific study on clinicopathologic findings of MCL here. MCL comprises approximately $4 \%$ of malignant lymphoma in the province of British Columbia [2] accounts for $6 \%$ of all NHLs in the USA [11] and 3.2\% of all malignant lymphoma in turkey [12]. In our study, MCL comprised about $4.57 \%$ of all malignant lymphomas. Median age is about 60 years [1], and in our study, it was 62 . The male to female ratio is more than $2 / 1$ [13], and in our study, it was 4/1. In one study which was done by Alexander J et al. in the USA [14], primary site of involvement in about $80.4 \%$ of patients was nodal, $3.6 \%$ hematologic, and $16 \%$ extranodal site, In

Table 3 Univariate and multiple Cox regression analysis

\begin{tabular}{|c|c|c|c|c|c|c|c|}
\hline \multicolumn{2}{|l|}{ Variable } & \multicolumn{3}{|l|}{ Univariate } & \multicolumn{3}{|l|}{ Multiple } \\
\hline & & Hazard ratio (HR) & $95 \% \mathrm{CI}$ for $\mathrm{HR}$ & $P$ value & Hazard ratio (HR) & $95 \% \mathrm{CI}$ for $\mathrm{HR}$ & $P$ value \\
\hline \multirow[t]{2}{*}{$\mathrm{Ki}-67$} & $<30$ & 1 & & & 1 & & \\
\hline & $\geq 30$ & 16.002 & $1.99-128.99$ & 0.009 & 14.71 & $1.65-130.88$ & 0.016 \\
\hline Age & & 1.11 & $1.05-1.17$ & $P<0.001$ & 1.12 & $1.03-1.22$ & 0.009 \\
\hline \multirow[t]{2}{*}{ Gender } & Male & 1.21 & $0.34-4.24$ & 0.77 & & & \\
\hline & Female & 1 & & & & & \\
\hline \multirow[t]{2}{*}{ Location } & Nodal + extra nodal & 0.995 & $0.32-3.09$ & 0.99 & & & \\
\hline & Leukemia & 1 & & & & & \\
\hline \multirow[t]{2}{*}{ Cytomorphology } & Classic + small & 0.6 & $0.13-2.7$ & 0.51 & & & \\
\hline & Blastic & 1 & & & & & \\
\hline
\end{tabular}




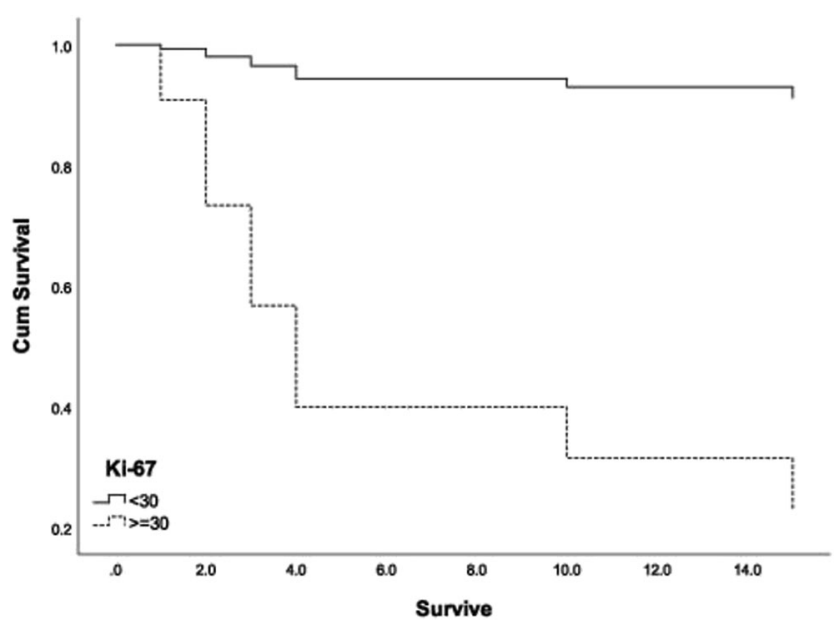

Fig. 2 Survival plot for multiple Cox regression of Ki-67 with adjusting on age variable

another study by Preetesh Jain and Michael Wang which published in American Journal of Hematology as 2019 update of MCL, leukemic non-nodal MCL was seen in 10 to $20 \%$ MCL patients[15], while in our study, patients presenting with leukemic non-nodal type were $28 \%$ which shows a difference in organ involvement at presentation probably due to effect of genetic and environment on tumor identity. Even though patients with leukemic presentations have been reported with the indolent course and longer survival [1], in our study, no significant difference in survival between nodal and non-nodal MCL was seen. In this study, $20 \%$ of MCL did not express CD5, while in another study, about $5 \%$ of MCL lack CD5 expression [16] and all of them had classic morphology. It is worth to mention that all of the blastic or pleomorphic variants showed CD5 aberrant expression. So irrespective of CD5 status, checking cyclin D1 expression is highly recommended on all low-grade B cell lymphoma (other than well-known entities such as small lymphocytic lymphoma, follicular lymphoma, and hairy cell lymphoma) and also on all high grade and pleomorphic ones when CD5 is positive. Sixty percent of

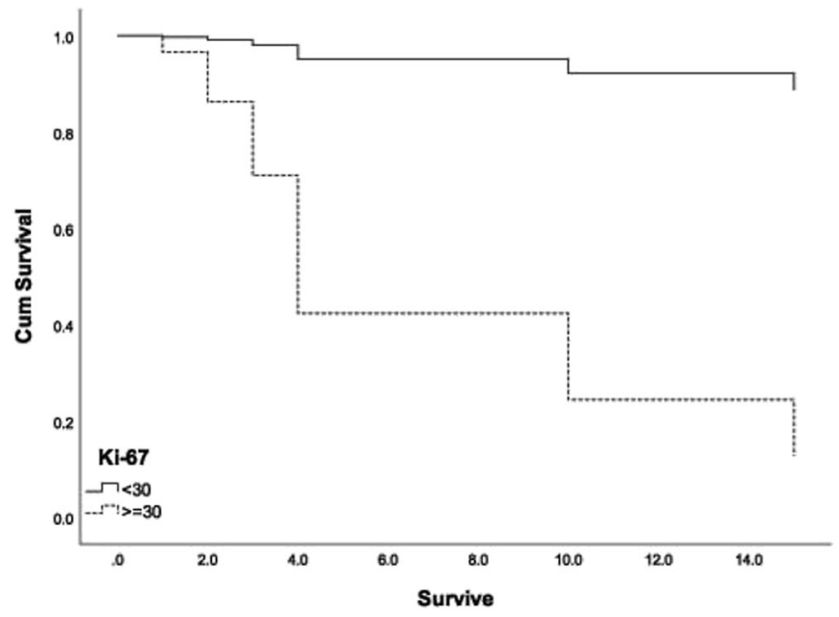

Fig. 3 Survival plot for univariate Cox regression patients with nodular histomorphology showed follicular dendritic cell meshwork expansion on immunostaining which propose follicular colonization of neoplastic cells in the germinal center while follicular dendritic cell meshwork expansion was not seen in other histoarchitectural of lymph node or extranodal sites.

\section{Conclusion}

Although MCL is an aggressive lymphoma, behavior varies from case to case from indolent with no active indications for treatment and can be watched initially, to very aggressive which needs various strategies of therapy according to risk stratification $[17,18]$ so the approach to a patient with MCL and protocol of therapy is different. Some clinicopathologic findings may predict an outcome.

In our study, although some results were not reliable and were limited due to low sample size (cytomorphology groups of pleomorphic and blastic type and histomorphology group of mantle zone pattern like) but Ki-67 and age were significant risk factors for the event (death), so these data may somehow help clinician predicting survival or even treatment regimen. In order to prevent overlooking of CD5-negative and blastic variants of MCL, we recommend cyclin D1 immunostaining on all low-grade B cell lymphoma and CD5-positive highgrade B cell lymphoma.

Funding information The study had been conducted by self-budget.

Data availability Database is available via corresponding author.

\section{Compliance with ethical standards}

Conflict of interest The authors declare that they have no conflict of interest.

Ethical approval This article does not contain any studies with human participants performed by any of the authors.

Informed consent Informed consent was obtained from all individual participants included in the study.

\section{References}

1. Swerdlow SH (2017) WHO classification of tumours of haematopoietic and lymphoid tissues. International Agency for Research on Cancer

2. Argatoff LH, Connors JM, Klasa RJ, Horsman DE, Gascoyne RD (1997) Mantle cell lymphoma: a clinicopathologic study of 80 cases. Blood. 89(6):2067-2078

3. Gao J, Peterson L, Nelson B, Goolsby C, Chen Y-H (2009) Immunophenotypic variations in mantle cell lymphoma. Am J Clin Pathol 132(5):699-706 
4. Dreyling M, Hiddemann W, Network EM (2009) Current treatment standards and emerging strategies in mantle cell lymphoma. ASH Educ Program Book 2009(1):542-551

5. Cohen JB, Zain JM, Kahl BS (2017) Current approaches to mantle cell lymphoma: diagnosis, prognosis, and therapies. American Society of Clinical Oncology Educational Book. 37:512-525

6. Geisler $\mathrm{CH}$, Kolstad A, Laurell A, Räty R, Jerkeman M, Eriksson M, Nordström M, Kimby E, Boesen AM, Nilsson-Ehle H, Kuittinen O, Lauritzsen GF, Ralfkiær E, Ehinger M, Sundström C, Delabie J, Karjalainen-Lindsberg ML, Brown P, Elonen E, for the Nordic Lymphoma Group (2010) The Mantle Cell Lymphoma International Prognostic Index (MIPI) is superior to the International Prognostic Index (IPI) in predicting survival following intensive firstline immunochemotherapy and autologous stem cell transplantation (ASCT). Blood. 115(8):1530-1533

7. Elaine Jaffe DAA, Campo E, Harris NL, Quintanilla-Fend L (2017) Hematopathology. Elsevier

8. Shah BD, Martin P, Sotomayor EM (2012) Mantle cell lymphoma: a clinically heterogeneous disease in need of tailored approaches. Cancer Control 19(3):227-235

9. Monabati A, Safaei A, Noori S, Mokhtari M, Vahedi A (2016) Subtype distribution of lymphomas in South of Iran, analysis of 1085 cases based on World Health Organization classification. Ann Hematol 95(4):613-618

10. Binesh F, Akhavan A, Behniafard N, Atefi A (2014) Clinicopathologic and survival characteristics of childhood and adolescent non Hodgkin's lymphoma in Yazd, Iran. Asian Pac J Cancer Prev 15(4):1585-1588
11. Mozaheb Z, Aledavood A, Farzad F (2011) Distributions of major sub-types of lymphoid malignancies among adults in Mashhad, Iran. Cancer Epidemiol 35(1):26-29

12. Zhou Y, Wang H, Fang W, Romaguer JE, Zhang Y, Delasalle KB, Kwak L, Yi Q, du XL, Wang M (2008) Incidence trends of mantle cell lymphoma in the United States between 1992 and 2004. Cancer: Interdiscip Int J Am Cancer Soc 113(4):791-798

13. Sağlam A, Esin E, Hayran M, Boyraz B, Üner A (2018) Distribution of lymphomas in Turkey: data of 4239 cases from a single institution using the WHO classification. Turkish journal of medical sciences. 48(5):1013-1023

14. Swerdlow SH, Williams ME (2002) From centrocytic to mantle cell lymphoma: a clinicopathologic and molecular review of 3 decades. Human pathology 33(1):7-20

15. Jain P, Wang M. Mantle cell lymphoma: 2019 update on the diagnosis, pathogenesis, prognostication, and management. American Journal of Hematology banner. First published: 08 April 2019 https://doi.org/10.1002/ajh.25487

16. Ambinder AJ, Shenoy PJ, Nastoupil LJ, Flowers CR (2013) Using primary site as a predictor of survival in mantle cell lymphoma. Cancer. 119(8):1570-1577

17. Rule S (2019) The modern approach to mantle cell lymphoma. Hematological oncology. 37:66-69

18. Kwatra KS, Paul PA, Dhaliwal D, Calton N, John JM (2016) Mantle cell lymphoma and variants: a clinicopathological and immunohistochemical study. Int J Sci Study 3(12):162-168

Publisher's note Springer Nature remains neutral with regard to jurisdictional claims in published maps and institutional affiliations. 